\title{
MHC class II-assortative mate choice in European badgers (Meles meles)
}

\begin{abstract}
The major histocompatibility complex (MHC) plays a crucial role in the immune system, and in some species, it is a target by which individuals choose mates to optimize the fitness of their offspring, potentially mediated by olfactory cues. Under the genetic compatibility hypothesis, individuals are predicted to choose mates with compatible MHC alleles, to increase the fitness of their offspring. Studies of MHC-based mate choice in wild mammals are under-represented currently, and few investigate more than one class of MHC genes. We investigated mate choice based on the compatibility of MHC class I and II genes in a wild population of European badgers (Meles meles). We also investigated mate choice based on microsatellite-derived pairwise relatedness, to attempt to distinguish MHC-specific effects from genomewide effects. We found MHC-assortative mating, based on MHC class II, but not class I genes. Parent pairs had smaller MHC class II DRB amino acid distances and smaller functional distances than expected from random pairings. When we separated the analyses into within-group and neighbouring-group parent pairs, only neighbouring-group pairs showed MHC-assortative mating, due to similarity at MHC class II loci. Our randomizations showed no evidence of genomewide-based inbreeding, based on 35 microsatellite loci; MHC class II similarity was therefore the apparent target of mate choice. We propose that MHC-assortative mate choice may be a local adaptation to endemic pathogens, and this assortative mate choice may have contributed to the low MHC genetic diversity in this population.
\end{abstract}

Keyword: Genetic compatibility hypothesis; MHC-assortative mating; Major histocompatibility complex; Pre- and postcopulatory mate choice; Sexual selection 